\title{
The Concept Study of Knowledge Value Added in Museum Digital Archives
}

\author{
CHENG-WEI FAN $^{1 *}$, YU-HUNG WANG $^{2}$ \\ ${ }^{1}$ Department of Digital Media Art, School of Art and Design, Fuzhou University of \\ International Studies and Trade \\ ${ }^{2}$ Division of Technology Education, National Science and Technology Museum \\ 28 Yuhuan Rd., Shouzhan Town, Changle City, Fuzhou, Fujian, CHINA
}

\begin{abstract}
Museum digital collection should explore its past (object digitization), present (cultural relic interpretation) and future (knowledge value-added and management) practices and benefits. Therefore, it needs a new perspective. Its importance and the preservation and reuse of Museum digital resources are worthy of attention. Looking at the development of museums, when modern culture has rapidly progressed from analog to digital media, from atoms to the world of bits, the role of museums in collecting objects has changed accordingly. The technology of information communication will become more important for the creation, sharing and preservation of Museum cultural resources through the reproduction and reproduction of exhibits. Based on the basic concepts of digital collections, this study aims to provide a clear direction for the development of Museum digital collections by clarifying the concept of knowledge added value.
\end{abstract}

Key-Words: Digital Collection, knowledge value-added, Digital Resources

Received: April 26, 2020. Revised: December 10, 2020. Accepted: December 17, 2020.

Published: December 23, 2020.

\section{Introduction}

The English word "Museum" is derived from the Greek word Mouseion, which is the temple of Muses, the goddess of art and Science in Greek mythology, for believers to dedicate. The early European and Asian museums are derived from the private collections of individuals or institutions, and the Ennigaldi-Nanna's museum is thought by some historians to be the first museum, although this is speculative. It dates to circa $530 \mathrm{BCE}$ [1]. A museum is an institution that collects, studies, exhibits and educates all kinds of things in various disciplines. It is concerned with both the natural world and the man-made things. The field of museums actually extends to many categories such as art, history, culture, Industry technology, religion, sports, biology, music and so on. It is both educational and entertaining, and can be visited by scholars and the general public at the same time. Through the detailed and reliable collection of cultural relics and the attraction of visual, auditory, tactile and other senses, museums can inspire thinking, stimulate emotions and improve the quality of human life.

From the premise that human society is constructed on the basis of power distribution and interest control, communication is no longer limited to the simple and equal relationship between people which to express feelings and enhance understanding. It often becomes the chip and result of power competition and interest exchange. Therefore, those who control and monopolize the dissemination of information are often the party with the greatest power and the strongest economic power in society. The museum has always been the place where a country's culture is inherited and knowledge is gathered. Therefore, from the perspective of communication, controlling and effectively providing the content of the Internet should be the key to the dominant position in the network communication, which is particularly important for the management of a nation and the preservation of its culture. Therefore, the research and development of museums has been an important project in various countries over the years.

\section{Related Literature}

\subsection{The trend of museum digitalization}

One of the most important developments of modern museums is the gradual shift from the focus of collection and preservation to the emphasis on educational functions and the improvement of audience awareness in management. Compared with the traditional museums which focus on the collection and preservation of cultural relics and the exhibition design of professional views, modern museums gradually begin to care about "what do the audience do in the process of museum visit?"? "And" what are the gains after the visit? In recent years, many science museums have not adapted the concept of the overall story of museum exhibition designing method, and started with the subject concept design method, that is, in the early stage of exhibition design planning, the division is carried 
out to classify similar exhibition contents and forming a whole story in the gallery, and now the designer in the museum intend to plan the existing exhibits which integrated into the configuration of the area, and each unit is set up by space during construction. Scharlors An X. etc., think that the framework is significant for rearranging the role of knowledge management in collaboration to protect the value of national archives resources as the knowledge asset of a state. It can reengineer the knowledge management activities in communication to share the value of national archives resources as the knowledge asset of a society. Furthermore, the framework can reconfigure knowledge management artifacts in connectivity to increase the value of national archives resources as the knowledge asset of the public [2]. The design and production of manufacturers as the object of the separate implementation, and finally the exhibitions are installed into the gallery. What is involved is the change of the exhibition education mode of Science Museum, and take the exhibition itself as a living teacher. Through the exploration and interaction of the audience on the exhibits, the audience's interest and motivation of self-learning are aroused, so as to achieve the purpose of sharing and creating knowledge in science museum. The exhibition concept of this system takes exploration as the major goal, and achieves the goal of self-learning through the process of the audience's exploration. The San Francisco Exploratorium museum is the best representative of this kind of exhibition concept. For different groups of visitors, it is found that there are obvious differences in the learning mentality of museums. For family groups, the main purpose of visiting museums is to learn new knowledge and education, while the purpose of school visits or tours is for entertainment. Visitors of these two groups can find that family groups spend more time in each exhibition. It shows that the exploration unit carries on the operation study, while the school's off campus visit or tour group tends to take a casual look at the flowers. Therefore, in order to enhance the function of museums, museums should not only attract the interest of museum visitors by means of exhibition and educational activities, but also make good use of digital resources to provide opportunities for further learning after visiting museums.

Observing the progress of civilization in various countries, we can know that the technology of knowledge transmission and reproduction is an important key. Therefore, with the rise of this wave of Internet technology, some scholars think the development of technology as the fourth wave of information revolution. In particular, this wave of information technology progress has found that many human life styles and social patterns been changed. The rise of technologies such as Email, MSN, Yahoo Messenger, Skype, Facebook, Instagram, Line, Wechat, Taoboo, Amazon, Google, YouTube e.t.c have sprung up and make the communication between people more and more rapidly and conveniently, and the information transmission is more and more faster, almost unlimited. Due to the rising number of networked technology, the concept of global village has become a world trend. The close communication between countries has become more complicated than ever before. A lot of social connecting activities of the new generation are formed in the Internet community, and knowledge sharing is not so far away and people can acquire knowledge from the online community. Therefore, how to cultivate the ability of the next generation to acquire knowledge for value-added creation in museums is very important issue. In Taiwan primary and secondary education system, the education of science and art has never really been implemented in our everyday life. The main problem is that most of the Taiwan school education system could not avoid the pressure of examination for the study under the high school period, and the competitive pressure of passing public examination to apply for better universities makes school teachers still unable to avoid the traditional teaching method to enhance their competitiveness. The modern museum should start to think about how to provide the visitors with an friendly and spontaneous learning environment, to inspire the children establishing a scientific research attitude from everyday life, to provide our children with the exactly learning methods, and lead them finding out their own confidence and happiness of learning, and expanding to long-life learning skills and abilities, so as to establish a good foundation for education in the next century.

\subsection{The Conception of Digital Collection in museum}

The ultimate value of museum collections is nothing more important than presenting museum collections to the public, transmitting the responsibility of Museum social education through exhibition, providing opportunities for people to entertain and learn, so as to obtain the public's recognition of the museum. As for the digital collections of museums, where are the benefits? Why do museums spend so much cost on digitizing their collections? The reason is for museums, digital 
collection is not only the digitization of museum collections but providing the knowledge of collection background. If the digital collection information provided by the museum is only the unorganized background information of relevant people, events, times and places, which is meaningless and of no value to the general public. It is only a raw data and without knowledge value. For example of Leonardo da Vinci's work- Mona Lisa Painting, it is very difficult for us to understand the connotation, spirit and beauty of this painting without the explanation of the background story. Therefore, how to interpret these unprocessed raw data into useful information for museums by mean of value-added research is a subject to be considered. Taking the Science and Technology Museum in Taiwan as example, the Museum's collection includes printed cultural relics, measuring instruments, telecommunication industry and Ancient key and lock relics etc. The ultimate goal of the museum is to be Taiwan's scientific and technological relics and industrial technology related collections. The collection policy include two points. One is to establish comprehensive collections policies in order to collect, purchase and maintain relics of technology. Another is to increase channels for utilizing museum collections and digitize relics of technology. In particular, Taiwan's rapid economic development during the Japanese occupation period and after the restoration which has left many important objects and historical documents in Taiwan's economic development. It's an opportunity for museum to collect and preserve scientific and technological relics. Through the accumulation of digital collection resources and reconstruction of metadata of these relics, it will be possible to preserve Taiwan's important industrial historical sites and expand the museum collection resources. However, how can we accumulate the resources of the museum's digital information? In addition to depend on museum's professional research, due to the limitation of research man-powers within the museum, they need to consider how to expand the resources and scope of value-added applications of digital collections through cooperation and participation with external institutions such as scholars, teachers and the public. In order to achieve this goal, the museum need to think about how to build a digital collection network of Taiwan's scientific and technological relics through the concept of knowledge sharing online and the data of digital collections. For a digital collection database, in order to bring its benefits into full play, it is important to record the knowledge of predecessors and transform it into public intellectual property of museums. Under this framework, knowledge value can be collected, exchanged, copied and shared. In the past, before the rise of information technology, human reproduction and sharing of knowledge were often limited to printed matter. The Internet makes it easier to share knowledge online. Therefore, digital collection is a new concept emerging in this framework. Basically, the collections of museums were carried out in closed ivory towers during the past, which could not be seen by the public. With the advance of the computer, museums start to set up the text information of the labels and cards on the computer database for the collection request. The rise of website has inspired the cooperation between different museums more and more close. Now museum can exchange and share the collection materials with each other through the integrated database system and has expanded to cross-border cooperation. In the future, Taiwan's museums must also have to strengthen their own research energy through cross-border cooperation. For example, the study of species can be able to quickly search for the relationship between different area species through the sharing and cross comparison of databases. In addition, the school teachers who teaching nature science can also access the databases of birds' collected singing which is established by the Museum of Natural Science to help their teaching, they can use this information to teach students how to listen and identify birds' calls and their habits, and how to apply the digital collection resources of such museums to education. I believe that will help and bring more impact ideas to the existing education system in Taiwan.

For the digital collection of museums, its connotation is a concept of knowledge sharing and value-added, that is, the concept of knowledge economy. Through the interface of digital collection, museum's researchers inside and outside can continue to accumulate and promote the research results. In particular, the development of the internet has made knowledge digitalized and spread rapidly. Therefore, the ability of applying information has become an important factor for museums to enhance knowledge sharing more effectively and economy. Does the museum collections need to be digitized? This is an issue which often discussed by the museum community. In terms of the use of database, digital information has the knowledge value of sharing, creating and reusing. For example, the Taiwan Paper Retrieval System which constructed by the National Library in Taipei is an indispensable reference database, 
because it can provide quick and accurate retrieval and query of Chinese papers and periodicals from the digital library. At the same time, it can also provide online access and printing through the cooperation of cross library. It also enhances the power of research by quickly accessing the required paper information and carry out value-added knowledge research activities. Except the purpose of management, the most important issue for the museum's digital collection is to create the value of knowledge sharing and how to turn the resources of museum into knowledge content that can be accessed by the public, and then add value to create its knowledge economy.

\section{The concept of value-added knowledge in digital archives}

The development of digital contents in Taiwan has lasted for several decades leading to accumulation of diverse and rich resources of digital contents. How to apply such resources in a value adding way to correspond to the ideas of knowledge reconstruction and knowledge-based economy is the key issue to be explored in this research [3]. The scharlors Roknuzzaman etc. finds some significant overlaps between digital library and knowledge management and argues that a generic knowledge management process of acquisition, organization, storage and retrieval, and dissemination of knowledge with receiving feedbacks can suitably be fitted in digital library. Thus an integrated digital library system can be consisted of digital resources, technological infrastructure, experience and expertise, digital library services and a knowledge management process. The integration of knowledge management can add value to developing a knowledge- based culture, management of intellectual assets, promotion of knowledge sharing, innovations in digital library services and a strong leadership position for digital library [4]. What is the value of the information behind the digital collections? Because information is not only information, it is the accumulation of important assets in the process of human knowledge forming, and the data needs to be transformed to be more readable and reliable. However, in the process of knowledge transformation, human beings must face two problems: one is how to find the information exactly and effectively. Another is how to analyze the information and transform it into a knowledge unit structure for application. The issue of searching information is an old problem. After the invention of printing, it had a new form. Printing scholars try to simplify the problem or make it more conveniently. Books make many items of information easier to find, but people need to find the right books for the first step. The rise of internet technology makes it easier to access and copy information. However, how to find the right and needed information in the internet world and transform it into knowledge is another problem to be solved. In the advanced society, knowledge and information are the main sources of productivity and growth. The concept of digital database was carried out in museums and libraries before the development of the internet. However, its system was limited to the organization and could not be accessed outside and carry out the concept of value-added and sharing of digital collection information. The rise of the internet enhances the accumulation and sharing of knowledge. However, if the mass knowledge database does not have a system structure that makes it easy for people to access and reuse, the information is a non-value database. Recently, the main problem of internet information is that it is not an encyclopedia like interface with strict classification structure and easy query and utilization. Therefore, the exactly and effective classification of digital information will be the problems to be solved in knowledge value-added. At present, many countries are studying the relevant solutions. The main solution to these problems is how to construct a simple and effective coding system for this database. Under this coding system, everyone can quickly quire his needs through the authorization metadata.

When constructing the digital collection, the digital resources at museum must be first organized, arranged, interpreted, and presented. On the web there are currently two ways to present the digital resources in Taiwan: (1) to activate the search engines for automatic sorting; (2) To record the traditional database, e.g. the widespread MARC format, by the catalogers in a library. Neither of them is good for application. Although the former search is enormous, but there's too much un-useful data. The standard of recording data in the latter method is too complicate, which makes difficult access for non-professionals and the speed of data recording lags behind the increasing resources. The entities of a great number of digital resources can be presented in the forms of words, images, sounds, animations, films, and virtual realities through txt, doc, pdf, gif, jpg, html, asp, mp3, wmv, rm, which possess diversity [5]. Due to the demand of precise retrievals and efficiency, people hope to have the standards for post recording of data set so that there are quick and convenient descriptions of the digital resources. Foreign museums and libraries, therefore, have had the standards set according to the different demands of the collected objects; some of which are DC (Dublin Core) [6] [7], CDWA (Categories for the 
description of works of Art) [8],CIDOC

( Documentation Committee of the International Council of Museum ) [9], VRA (Core Categories for Visual Resources ) [10], EAD (Encoded Archival Description) [11], MARC (Machine Readable Cataloguing) [12], and ECHO (European Chronicles On-line) [13].

The evolution of information science and photoelectric technology has been oriented in the development of hardware, especially in modular system. The elements for the integration of conductive circuits, electromechanical devices, and photoelectric transformations have been designed in the system modules of products. The interfaces between systems have adopted international standards for compatibility and common applicability. The development of software, e.g. object-oriented and JAVA elements, is also in the similar direction with the emphasis on the compatibility of cross-platforms and systems, and exchangeability. The purpose is to set the foundation for system integration by boosting efficiency and reducing system costs, and thus become beneficial for system upgrades and revisions. According to many researchers, the current web-based educational tutorials are generally poor in educational content. The World Wide Web is a relatively new technology and the early adopters were individuals skilled in programming and HTML skills, but not necessarily knowledgeable about educational concepts [14].

On the Internet, the traditional communication barriers between people, such as time, place and to an extent social status, are no longer existed. The power to reach people and access information together with other characteristics, such as presenting and receiving information in multimedia format, makes the Internet perfect for a constructivist to create his/her teaching environment [15]. Based on the same concept, a museum must consider which system structure is needed for undertaking the project of digitalizing its collections. The issue of whether data integration can be applied to demonstrate the core of the museum collection and research, expansion of the scope and contents of the value-added services, must also be considered. The three basic demands of the digital contents are as follows:

(1) The demand of the management: With the aim of collection management, the secured management of the information of the collected objects, the establishment of the basic information, the management of the information of the maintenance for the collected objects, and the management of the information of demand for exchange and borrow between museums.

(2) The demand of the researchers: With the aim of the research of the collected objects, the consultation, proofreading and corrections of the basic information, the interpretations of the contents and related references and literature of the collected objects are the concerned issues.

(3) The demand of the general viewers: The viewers are concerned with the gain of the knowledge in regard to the history, theory, application and impact of the collected objects. Among the different demands of the system structures, the format of the basic functions and the establishment of the integration of the intellectual contents of the system must be considered first.

According to different metadata standards, the basic functions of a collected object includes [16] [17]:

(1)The research, exhibition, and educational functions of objects.

(2)The recording and management demand of objects.

(3) The interactions of objects among museums.

(4) The interactions of objects among different social groups.

The basic structure of the database for the digital collection of an object must be open and flexible to expand and interact with other museums. Presently, HTML (Hyper Text Markup Language), SGML (Standard Generalized Markup Language), and XML (Extensive Markup Language) are the three available structures. Although, HTML is easy to start and develop, it can not label itself to describe more complicated document structures or design more precise web inquiries, which makes it an unsuitable tool to be the core developing program for digital collection. SGML is highly flexible and is able to describe the structure of any information or complicated documents. It can also cross platforms and allow the information to be reused. But its standard specifications are extremely complicate and the development costs are too high. As a result, libraries and museums tend to adopt the improved SGML, and take XML as the markup languages in the development of the technology for electronic information exchange.

Then, how to add value to the knowledge of digital collection? It can be discussed from several aspects.

\subsection{From the transfer and construction of knowledge:}

according to professor $\mathrm{Gu}$ [18] proposed on the four civilizations of library, the evolution process of library in human knowledge civilization can be divided into four stages, namely: (1) memory storage of knowledge on the media; (2) reproduction, 
circulation and transmission of knowledge media; (3) reference and referral service of knowledge content; (4) knowledge connotation and knowledge Performance management. The scholar Wu Sihua [19] once proposed three mechanisms for the construction of knowledge society including knowledge creation, knowledge circulation, and knowledge value-added. In terms of knowledge value-added, he thinks that knowledge needs a value-added process to be successful commercialization and bring customer value. It can be seen from the development of knowledge transfer and construction that the concept of knowledge value-added of digital archives in the future should be based on the development of personalized knowledge construction management system, that is, how to construct personal knowledge base and knowledge network, and provide management services for creating personal knowledge innovation and production environment.

\subsection{From the value-added application direction of digital collection industry:}

Professor Xiang J. etc. think from the perspective of value-added application of digital collection, and regard the digital content industry as three categories: leisure and entertainment industry, education and learning industry, culture and life industry. As for the content of application products, the value-added products of digital collections in Taiwan include: making various databases (images, electronic publishing, art, etc.), or converting digital collections into various thematic products according to the need of customers, such as virtual reality games, interactive teaching $\mathrm{CDs}$, 3D films, 3D object navigation, etc. Some manufacturers provide customers with the opportunity to create their own value-added digital collection materials. For example, they can create various interesting finished products such as E-cards and personal web pages for download and edit the digital archive image and video files by cellphone to turn them into digital image congratulations and screen protectors[20] [21].

\subsection{From the perspective of digital collection business application model:}

The files and materials after digital archives can be used for learning, research, and economic equivalent through value-added application, and then play the true purpose of digital archives. The scope of application of digital collections is quite extensive. Professor Xiang J. etc. [22] have divided the business models of digital collection industry application into five categories: (1)Industrial value-added material library; (2)Integrated products; (3)Cultural value-added products; (4)Product added content services; (5)Digital technology software and services; they think that digital "materials" should be used, combined with the existing or newly developed "platform tools", such as technology development and upgrading, change of user interface and habits, and "customer service mode" such as authorization mode, an operation system can effectively release digital resources from digital materials through the resource conversion process in the business value chain, so as to realize the purpose of sustainable operation.

So, in summary of above research, the museum of digital collections can be applied to "leisure and entertainment", "Learning and Research", "Culture and Life". Among them, the "Culture and Life" field is the application of digital collection resources that are closely related to the life of the general public. Literary creations through digital media, and cultural materials integrated into a database (such as art, photos, images, etc.), not only extract elements from it. When added value of knowledge in digital archives, we can make into people's livelihood products (such as reproduction of paintings, ceramics, textiles, stationery, accessories, etc.), and can be combined with the context of in-depth tourism for cultural guided tours.

\section{Summary}

The museum's four major functions of collect, preserve, interpret, and display objects of artistic, cultural, or scientific significance for the education of the public are used to examine the development and application of the museum's digital collection. The museum's digital collection is intended to present and preserve the original content and exhibits in a digital way to achieve sustainable operation and the purpose of preserve for human spiritual needs. The main development is focusing on a professional database query system, which is still produced by processing, compiling or sorting the original relic or digital data of the original collection of museum, and integrates it into the museum's digital learning system, supporting museum's exhibitions and science education activities. Its ultimate goal is for the concept of lifelong learning in museums, as shown in Fig. 1. Therefore, how to satisfy the personal knowledge construction management system, organize personal knowledge base and knowledge network, and then carry out the management service of production environment and creating personal knowledge innovation in the future, will be the most important key concept of knowledge added value. 


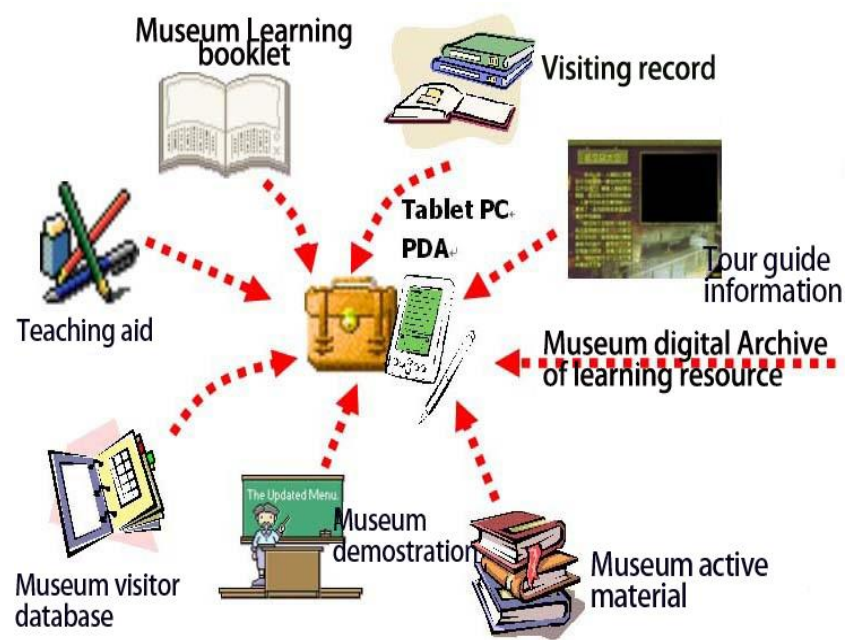

Fig. 1 The Architecture of Mobile Learning System in the Museum

This paper attempts to describe the future needs from an imaginable view. The "Printing" search is added to the function of the internet search engine. Each caption of data contains the interpretation metadata of people, events, time, place and object, as well as a list of knowledge structures. If you want more detail information, you can type more content like moveable typesetting and Bi Sheng; Then you can find more structure information about the relationship of printing, moveable typesetting and $\mathrm{Bi}$ sheng. When you are a teacher who need these information for teaching. You can access any relatively printing data format, including textbook, PPT document, video teaching material etc., and the software will help you to make more accurate query and then you can combine those as knowledge unit. If this knowledge unit need to be used as a student's textbook, you need more the format of picture explanation, film explanation and even animation explanation, which can be found immediately through the knowledge metadata. Through different formats of knowledge metadata units, we can quickly combine the desired digital teaching materials. The diverse application of new technologies has changed how museums communicate with their visitors pivoting museums from an "education" orientation to a "learning" orientation. The importance and complexity of learning tools has also increased. Education in museums focuses on providing the visitor a true to life scenario or actual participation in activities to facilitate the learning of knowledge or skills; therefore, early tour methods consist of pamphlets, guided explanations, and listening devices which have evolved to the mobile devices of today. The medium used in museum tours and education activity has evolved from still to interactive multimedia. Computer multimedia surely plays an important role in museum tours and education but computers are cumbersome and not easily transported. Therefore, they have been replaced by light, mobile devices that are now used in museum tours and education. But there is more important is that the mobile device can easy access the museum digital archive of learning resource. What kinds of learning resource the museum should provide? It depends on how to design the knowledge metadata of museum and make it more friendly and useful. At the same time, the knowledge metadata can help to analyze the suitable age of such teaching materials, which teaching effect can be achieved, and the coherent teaching contents in the future. If such a knowledge metadata can be implemented, we believed that the application of knowledge value-added of digital archives will be a huge step for the development of human learning. By the way, if the knowledge metadata including artificial intelligence, the knowledge value-added application of Museum digital collection should be combined with the museum's collection research, exhibition, education, tour guide and audience community, and analysis to integrate and manage the metadata, so as to satisfy the personalized knowledge needs and achieve the goal of promoting Museum lifelong learning.

\section{Funding}

We gratefully acknowledge the financial support by the Fuzhou University of International Studies and Trade foundation of China.

\section{References:}

[1] Rodini, E., Preserving and Perpetuating Memory at the Musée Nissim de Camondo in Paris," Museum History Journal 7(1), 2014, pp. 36-54.

[2] An, X., Bai, W., Deng, H., Sun, S., Zhong, W. and Dong, Y., A knowledge management framework for effective integration of national archives resources in China, Journal of Documentation, 73(1),2017,pp. 18-34.

[3] Liao Tun-Ju., A Study on Developing Value-added Knowledge Model of Digital Archives by Applying Knowledge management An Example of 2D Animation Development of Sugar Refining Industry Culture, Journal of National Taiwan College of Arts Vol.100, 2017, PP. $59-87$.

[4] Roknuzzaman, Md., Kanai H., Umemoto, K., Integration of knowledge management process into digital library system: A theoretical perspective, Library Review, 58(5),2009, pp. 372-386.

https://doi.org/10.1108/00242530910961792 
[5] Clark, R.E., Reconsidering research on learning from media. Review of Educational Research, Vol 53(4), 1983, pp. 445-459.

[6] Kunze, J. (1999), Encoding Dubin Core Metadata in HTML, 2019/10/29 [cited; Available from: http://www.ietf.org/rfc/rfc2731.txt.

[7] Miller E., Miller P., and Brickley d. (1999) Guidance on Expressing the Dublin Core within the Resource Description Framework (RDF)., [cited 2019/10/31]; Available from: http://www.ukoln.ac.uk/metadata/resources/dc/da tamodel/WD-dc-rdf/.

[8] Murtha Baca and Patricia Harpring. (2006) CDWA List of Categories and Definitions. 2019/12/25 [cited; Available from: http://www.getty.edu/research/conducting research/standards/cdwa/categories.html.

[9] ICOM. ( 1995), CIDOC Guidelines for Museum Object Information. 2019/12/1 [cited; Available from:

http://www.willpowerinfo.myby.co.uk/cidoc/guid e/guideint.htm.

[10] VRA. (2002), VRA Core Categories, Version 3.0-a project of the Visual Resources Association Data Standards Committee 2019/12/1 [cited; Available from: http://www.vraweb.org/vracore3.htm\#core.

[11] EAD. (2002), Encoded Archival Description. 2019/10/31 [cited; Available from: http://www.loc.gov/ead/.

[12] Library of Congress. (2006), MARC Standards. 2019/12/1 [cited; Available from: http://www.loc.gov/marc/.

[13] ECHO. (2000), Metadata Format Definition and Acquisition. 2019/10/31 [cited; Available from: http://pc-erato2.iei.pi.cnr.it/echo/workpackages/w p3.html.

[14] Murray, T.. From Story Boards to Knowledge Bases, the First Step in Making CAI 'Intelligent'. In Carlson \& Makedon (Eds.) Educational Multimedia and Hypermedia, Proceedings, 1996, pp. 509-514. Charlottesville VA: AACE.

[15] Janassen, D.H., Peck, K.L. \& Wilson, B.G. Learning with Technology: A Constructivist Perspective.Upper Saddigital librarye River, New Jersey: Prentice Hall, 1999.

[16] Chen Yaning, Chen Shujun, Metadata Development and Analysis in Digital Museums, Library Science and Information Science, 27(2), 2001, pp.52-66.

[17] Holmes, O. W., Archival Arrangement: Five Different Operations at Five Different Levels, American Archivist, 27(1), 1964, pp.21-41.

[18] Gu M., On the Four Civilizations of the Library, Journal of the Chinese Library Association, 73,
2004, pp. 21-26.

[19] Wu Sihua, Industrial Innovation in the Knowledge Century: Issues and Countermeasures, Science and Technology Development Policy Report SR8902, 2000.

[20] Xiang J., Chen X. H., Zheng D. F., Wei Y. H., Discussion on the Value-Added Application of Digital Collection, Journal of Library and Information Science, 2(1), 2003.

[21] Xiang J., Chen X. H., Wei Y. H., Gao S. X., A Preliminary Study of Creative Value-Added Application of Digital Collection, Journal of Information and Education, 91, 2002, pp.10-16.

[22] Xiang J., Chen X. H., Chen Z. Z., Guo Z. Y., Discussion on the Business Model of Digital Collection Industry, Journal of the Chinese Library Association, 2005.

\section{Creative Commons Attribution License 4.0 (Attribution 4.0 International, CC BY 4.0)}

This article is published under the terms of the Creative Commons Attribution License 4.0 https://creativecommons.org/licenses/by/4.0/deed.en_US 